\title{
A Single Gyroscope Can Be Used to Accurately Determine Peak Eversion Velocity during Locomotion at Different Speeds and in Various Shoes
}

\author{
Christian Mitschke * (D), Matthias Öhmichen and Thomas L. Milani \\ Department of Human Locomotion, Chemnitz University of Technology, 09126 Chemnitz, Germany; \\ matthias.oehmichen@s2008.tu-chemnitz.de (M.Ö.); thomas.milani@hsw.tu-chemnitz.de (T.L.M.) \\ * Correspondence: christian.mitschke@hsw.tu-chemnitz.de; Tel.: +49-371-531-32196
}

Academic Editors: Bjoern Eskofier and Sunghoon Ivan Lee

Received: 30 May 2017; Accepted: 23 June 2017; Published: 27 June 2017

\begin{abstract}
Gyroscopes have been used in previous studies to measure the peak angular velocity of the shoe or foot in the frontal plane (evVel). However, it is not clear whether different test conditions (footwear hardness or locomotion speed) can influence the accuracy of evVel. The purpose of the present study was to compare the accuracy of gyroscopes and electrogoniometers when measuring evVel and the time until evVel (t_evVel) in 12 different conditions using a single axis gyroscope attached to the heel cap. Twenty-four recreational runners were instructed to walk and run on a 15-m indoor track at four locomotion speeds $(1.5,2.5$, and $3.5 \mathrm{~m} / \mathrm{s}$, and individual running speed) and in three footwear conditions (low to high hardness). The gyroscope data and electrogoniometer data were sampled at a rate of $1000 \mathrm{~Hz}$. Comparisons between both measurement devices showed small mean differences up to $49.8 \pm 46.9 \mathrm{deg} / \mathrm{s}$ for evVel and up to $5.3 \pm 3.5 \mathrm{~ms}$ for t_evVel. Furthermore, strong relationships between gyroscope and electrogoniometer data were found for evVel as well as for $t$ _evVel for all conditions. It can be concluded that gyroscopes can be used to accurately determine evVel and $t \_e v V e l$ under a variety of conditions.
\end{abstract}

Keywords: peak eversion velocity; gyroscope; electrogoniometer; running speed; footwear; material characteristics

\section{Introduction}

Foot pronation is a natural damping mechanism during walking and running, whereby the eversion - a part of pronation-plays a crucial role in reducing the acting forces on the lower limbs. Eversion velocity seems to be more decisive for critical loading than the maximum angular excursion. In this context, studies have reported that high eversion velocities could be associated with medial tibial stress syndrome [1-3]. Aside from investigating injuries, previous studies examined the influence of footwear construction or foot orthoses [1,4-7], exhaustion [8], and running pace [9] on maximum eversion velocity and the time until it occurs.

To investigate kinematics, optoelectronic systems (e.g., Vicon, Qualisys) have been established as the gold standard $[8,10,11]$. However, time-intensive preparation of subjects, considerable laboratory size, and high prices are the disadvantages of these systems. Furthermore, data capturing is restricted to indoor laboratory measurements. Low sampling rates between 50 and $500 \mathrm{~Hz}$ and marker-artifacts due to wobbling can also influence the accuracy of calculated parameters, especially when observing kinematics in the frontal and transversal planes.

In comparison, electrogoniometers are considered an easy-to-use alternative when investigating rearfoot motion in relation to the shank in the frontal plane $[5,10,12]$. The electrogoniometer is attached to the heel cap of the shoe and at the posterior lower leg to accurately measure the rotation about 
the subtalar joint axis at distinctly higher sampling rates of up to $1000 \mathrm{~Hz}[5,12]$. The disadvantage of this system is the flexible arm between the shoe or foot and the attachment at the lower leg. During long-term measurements in the field, the system's mass can influence walking and running gait. Furthermore, material wear of the flexible arm connections can occur due to the continuous mechanical stress during prolonged runs.

The third option is to use micro-electromechanical sensors (MEMS). Wearable motion sensors (e.g., gyroscopes and accelerometers) are an inexpensive alternative to optoelectronic systems [13]. These sensors are simple to handle, cost-effective, and suitable for field measurements $[8,11,13]$.

A few studies investigated the accuracy of MEMS when comparing angles or angular velocities with optoelectronic systems $[8,10,14]$. When examining the agreement of the maximum angular velocity of the shoe in the frontal plane (eversion velocity), they found moderate [8], acceptable [10], and good [14] agreement between the systems. Investigated running speeds were in a narrow range between $3.2 \pm 0.4$ and $4.0 \pm 0.2 \mathrm{~m} / \mathrm{s}$. The influence of locomotion speed (walking or running) or footwear characteristics on the accuracy of the maximum angular velocity was not investigated. However, this information is necessary to determine whether differences in angular velocities between footwear conditions as well as between locomotion speeds are caused by measurement errors of the gyroscopes or by the investigated conditions themselves.

Therefore, the aim of this study was to compare the accuracy of a gyroscope and an electrogoniometer in quantifying peak eversion velocity (evVel) and the time until evVel ( $t$ evVlel). To avoid effects due to different sampling rates and marker-artifacts, the gyroscope data in this study were compared to the electrogoniometer data. Four locomotion speeds and three different footwear conditions were investigated. We hypothesized that evVel measured with a gyroscope is related to evVel measured with electrogoniometer (H1). Furthermore, we hypothesized that $t \_e v V e l$ with the gyroscope is related to $\mathrm{t} \_$evVel with the electrogoniometer $(\mathrm{H} 2)$.

\section{Materials and Methods}

\subsection{Participants}

Twenty-four recreational rearfoot runners (age: $24.7 \pm 4.1$ years; height: $178 \pm 9.7 \mathrm{~cm}$; weight: $74.0 \pm 6.0 \mathrm{~kg}$; running experience: $8.5 \pm 3.9$ years; training hours: $3.3 \pm 2.0 \mathrm{~h}$ per week) participated in this study. This study was approved by the Ethics Committee of the Faculty of Behavioural and Social Sciences of the university (V-103-17-HS-CM-Bodenkontakt-25082015). The participants gave written informed consent to their participation in the study.

\subsection{Test Procedure}

During the test, participants wore three different running shoes, which were provided by the laboratory in men's UK size 8: Adidas AdiStar (AD); Asics Gel Nimbus 12 (AS); PUMA Speed 600 (PU). After an individual warm-up and familiarization with the measurement setup, five repeated running and walking trials were recorded on a 15-m indoor running track at four different velocities $(1.5 \pm 0.1$, $2.5 \pm 0.1,3.5 \pm 0.1 \mathrm{~m} / \mathrm{s}$, and self-selected individual speed, close to their personal standard training velocity) in each footwear condition. The three footwear conditions and the four velocities were investigated in randomized order. Individual speed was in mean $3.63 \pm 0.3 \mathrm{~m} / \mathrm{s}$. Velocities were monitored using two light barriers placed eight meters apart.

\subsection{Experimental Setup}

To measure the angular velocity of the shoe in the frontal plane, a gyroscope (IDG-650, InvenSense, TKD, Tokyo, Japan, measurement range $\pm 2000 \mathrm{deg} / \mathrm{s}$ ) in a small box was attached to the heel cap of the right shoe. The positive peak eversion velocity (evVel_Gyro) was defined as the maximum positive angular velocity from the gyroscope signal during ground contact. 
As reference, a light-weight electrogoniometer (Megatron MP10) was attached to the heel cap of the right shoe (potentiometer), next to the gyroscope box, and at the posterior lower leg parallel to the Achilles tendon (flexible arm) to measure the rearfoot motion of the shoe in relation to the shank in the frontal plane $[5,12]$. The axis of rotation was adjusted to represent the approximate position of the subtalar joint axis [5,12]. A neutral rearfoot angle was individually defined for each subject in each footwear condition during a static trial on a calibration plate $[12,15]$. The angular velocity of the goniometer signal was obtained by simple numerical differentiation of the goniometer signal during ground contact. The maximum positive angular velocity was declared as evVel_Gonio. Furthermore, the elapsed time between initial ground contact and evVel_Gonio and evVel_Gyro were determined (t_evVel_Gonio and t_evVel_Gyro).

Gyroscope and goniometer operated synchronously and data were recorded at $1000 \mathrm{~Hz}$ by a data logger, secured in a waist belt. Data logger and PC were connected via a 10-m cable. To confirm rearfoot strike pattern, running was controlled visually according to Giandolini et al. [16]. The presence of the impact peak in vertical ground reaction data was checked. Ground reaction force measurements were simultaneously recorded using a force plate $(9287 \mathrm{BA}, 0.6 \times 0.9 \mathrm{~m}, 1000 \mathrm{~Hz}$, Kistler, Winterthur, Switzerland) that was synchronized with the gyroscope and electrogoniometer. According to Laughton et al. [17], the threshold of the force plate was set at $10 \mathrm{~N}$ to determine foot touchdown. To remove noise, raw data were filtered with a zero-lag Butterworth low-pass filter (gyroscope and goniometer: 4th order at $50 \mathrm{~Hz}$; force plate: 2nd order at $50 \mathrm{~Hz}$ ). Data post-processing was done using MATLAB R2016b (MathWorksTM, Natick, MA, USA).

\subsection{Footwear Conditions}

To quantify the differences of the materials of the rearfoot area, the three shoes were tested in a servo-hydraulic testing device (HC10; Zwick GmbH \& Co. KG; Ulm, Germany) as described in Schwanitz \& Odenwald [18] and Schwanitz et al. [19]. Each shoe was tested mechanically ten times. We analysed the heel height ( $\mathrm{Heel}_{\text {height }}$ ) and the stiffness in the rearfoot area (stiffness) between 1000 and $1500 \mathrm{~N}$ as described in Schwanitz \& Odenwald [18]. Footwear with high mechanical stiffness could be described as relatively hard, and footwear with lower mechanical stiffness as relatively soft.

\subsection{Statistical Analyses}

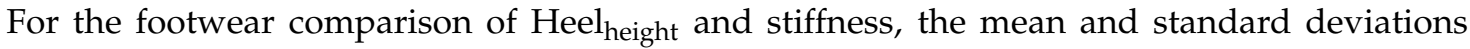
(mean $\pm \mathrm{SD}$ ) were calculated. Given that parameters were normally distributed according to the Shapiro-Wilk test, a one-way analysis of variance (ANOVA) followed by Bonferroni post-hoc tests were used to determine whether differences existed between mechanical footwear characteristics. Effect size (Cohen's $d$ ) was calculated to quantify the magnitude of differences between shoe characteristics. The coefficients were interpreted as trivial effect $(d<0.2)$, small effect $(d<0.5)$, medium effect $(d<0.8)$, and large effect $(d \geq 0.8)$ [20]. For the comparison of gyroscope and goniometer data, mean differences (MDs) and standard deviations (SDs) between evVel_Gonio and evVel_Gyro were calculated for evVel as well as for $\mathrm{t} \_$evVel. For evVel, a positive MD would indicate greater evVel measured by gyroscope, negative MD would indicate greater evVel measured by electrogoniometer. Furthermore, a positive MD of $t \_e v V e l$ would indicate that evVel was measured earlier with the electrogoniometer than with the gyroscope. Finally, evVel_Gyro was related to evVel_Gonio with correlation and regression analyses for evVel and t_evVel. Pearson's correlation coefficient was interpreted as trivial $(r \leq 0.1)$, small $(r \leq 0.3)$, moderate $(r \leq 0.5)$, large $(r \leq 0.7)$, very large $(r \leq 0.9)$, and extremely large $(r>0.9)$ [21]. Statistical significance was set at $\alpha=0.05$ for all statistical analyses. 


\section{Results}

\subsection{Footwear Characteristics}

ANOVA revealed that rearfoot stiffness and Heel height were influenced by footwear type, with all pair-wise comparisons reaching statistical significance $(p<0.001)$. Cohen's $d$ showed large effects in all pair-wise comparisons. The lowest rearfoot stiffness was found for AS $(156.9 \pm 0.1 \mathrm{~N} / \mathrm{mm})$ and the highest was found for $\mathrm{AD}(210.3 \pm 0.4 \mathrm{~N} / \mathrm{mm})$ (Table 1). Furthermore, the greatest Heel height was measured for AS $(30.4 \pm 0.0 \mathrm{~mm})$ and the lowest Heel $\mathrm{height}_{\text {was }}$ found for $\mathrm{AD}(22.7 \pm 0.0 \mathrm{~mm})$.

Table 1. Group mean \pm standard deviation (SD) for mechanical testing of the three footwear conditions (Adidas AdiStar (AD); Asics Gel Nimbus 12 (AS); PUMA Speed 600 (PU)) for rearfoot stiffness and heel height; statistical significance $(p<0.05)$ and large effects $(d \geq 0.8)$ were found between all shoe conditions for both parameters.

\begin{tabular}{ccccc}
\hline \multirow{2}{*}{ Footwear Condition } & \multicolumn{2}{c}{ Stiffness $(\mathbf{N} / \mathbf{m m})$} & \multicolumn{2}{c}{ Heel $_{\text {height }}$ (mm) } \\
\cline { 2 - 5 } & Mean & SD & Mean & 土SD \\
\hline AD & 210.3 & 0.4 & 22.7 & 0.0 \\
AS & 156.9 & 0.1 & 30.4 & 0.0 \\
PU & 177.8 & 0.4 & 24.2 & 0.0 \\
\hline
\end{tabular}

\subsection{Peak Eversion Velocity}

The largest evVel was found for the individual speed and the shoe with the hardest sole configuration and the lowest heel height (footwear AD: evVel_Gonio: $526.7 \pm 129.0 \mathrm{deg} / \mathrm{s}$; evVel_Gyro: $565.1 \pm 142.9 \mathrm{deg} / \mathrm{s} ;$ Figure 1). Generally, the comparison of evVel_Gonio and evVel_Gyro revealed an MD up to $49.8 \pm 46.9 \mathrm{deg} / \mathrm{s}$ (Table 2: footwear AD at $3.5 \mathrm{~m} / \mathrm{s}$ ). Larger evVel was found in nine conditions for evVel_Gyro. Only AS revealed lower evVel_Gyro for the three running conditions. Pearson's correlation coefficient showed very large positive correlations $(0.7<r \leq 0.9)$ for PU $(2.5$, $3.5 \mathrm{~m} / \mathrm{s}$ and individual speed), and extremely large positive correlations $(r>0.9)$ for all other conditions. The regression analysis revealed that evVel_Gonio was strongly related to evVel_Gyro at all velocities and all footwear conditions $(p<0.001)$.

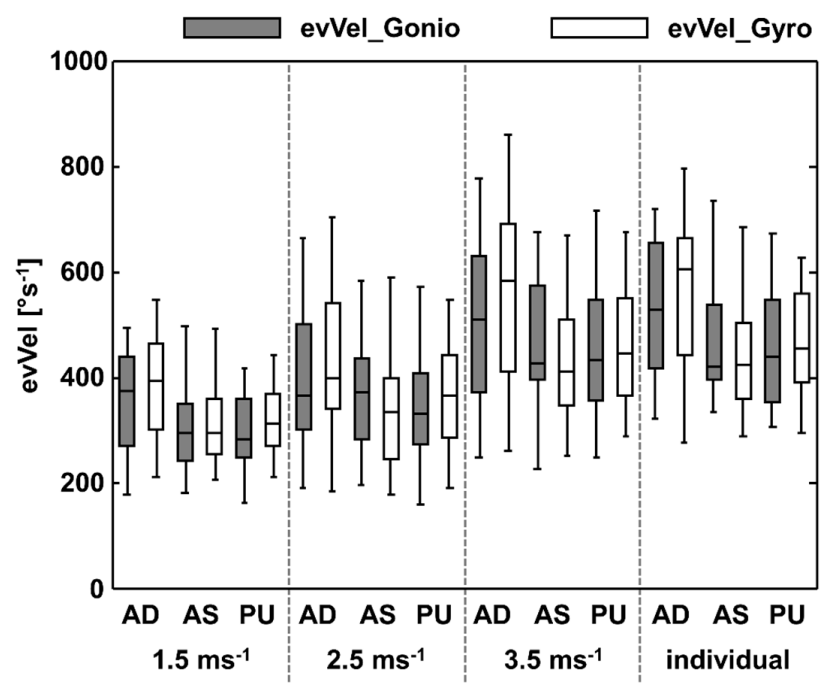

Figure 1. Boxplot of maximum positive angular velocity (evVel_Gonio, grey) and positive peak eversion velocity (evVel_Gyro, white) for the four velocities and three footwear conditions: Adidas AdiStar (AD); Asics Gel Nimbus 12 (AS); PUMA Speed 600 (PU). 
Table 2. Mean differences (MD) and standard deviations (SD) between evVel_Gonio and evVel_Gyro of the four velocities and the three footwear conditions: Adidas AdiStar (AD), Asics Gel Nimbus 12 (AS), PUMA Speed 600 (PU); Pearson's correlation coefficient was interpreted as trivial $(r \leq 0.1)$, small $(r \leq 0.3)$, moderate $(r \leq 0.5)$, large $(r \leq 0.7)$, very large $(r \leq 0.9)$, and extremely large $(r>0.9)$.

\begin{tabular}{cccccc}
\hline Speed & Footwear Condition & MD (deg/s) & SD (deg/s) & $\boldsymbol{r}$ & $\boldsymbol{R}^{\mathbf{2}}$ \\
\hline \multirow{3}{*}{$1.5 \mathrm{~m} / \mathrm{s}$} & AD & 23.7 & 55.9 & $0.96^{1}$ & $0.92^{2}$ \\
& AS & 10.4 & 49.3 & $0.94^{1}$ & $0.87^{2}$ \\
& PU & 26.2 & 50.8 & $0.91^{1}$ & $0.82^{2}$ \\
\hline \multirow{3}{*}{$2.5 \mathrm{~m} / \mathrm{s}$} & AD & 30.3 & 26.0 & $0.93^{1}$ & $0.86^{2}$ \\
& AS & -30.6 & 27.2 & $0.94^{1}$ & $0.87^{2}$ \\
& PU & 20.5 & 30.5 & $0.89^{1}$ & $0.79^{2}$ \\
\hline \multirow{3}{*}{$3.5 \mathrm{~m} / \mathrm{s}$} & AD & 49.8 & 46.9 & $0.97^{1}$ & $0.94^{2}$ \\
& AS & -30.9 & 35.8 & $0.91^{1}$ & $0.83^{2}$ \\
& PU & 12.3 & 45.0 & $0.88^{1}$ & $0.76^{2}$ \\
\hline \multirow{3}{*}{ Individual speed } & AD & 38.4 & 44.3 & $0.92^{1}$ & $0.84^{2}$ \\
& AS & -33.7 & 49.0 & $0.91^{1}$ & $0.81^{2}$ \\
& PU & 23.7 & 58.8 & $0.88^{1}$ & $0.77^{2}$ \\
\hline
\end{tabular}

${ }^{1}$ Pearson's correlation coefficient: $p<0.001 ;{ }^{2}$ Regression analyses: $p<0.001$.

\subsection{Time Until Peak Eversion Velocity}

Peak eversion velocity occurred between $13.6 \pm 4.5 \mathrm{~ms}$ (footwear AD: $3.5 \mathrm{~m} / \mathrm{s}$ ) and $27.8 \pm 7.6 \mathrm{~ms}$ (footwear AS: $2.5 \mathrm{~m} / \mathrm{s}$ ) after initial ground contact for evVel_Gonio, and between $16.8 \pm 3.4 \mathrm{~ms}$ (footwear AD: $3.5 \mathrm{~m} / \mathrm{s}$ ) and $29.4 \pm 7.3 \mathrm{~ms}$ (footwear AS: $2.5 \mathrm{~m} / \mathrm{s}$ ) after initial ground contact for evVel_Gyro (Figure 2). EvVel_Gyro was detected later than evVel_Gonio in all 12 conditions (Table 3). The comparison of t_evVel_Gonio and t_evVel_Gyro revealed an MD of up to $5.3 \pm 3.5 \mathrm{~ms}$ (footwear $\mathrm{PU}$ at $1.5 \mathrm{~m} / \mathrm{s})$. Pearson's correlation coefficient showed an extremely large positive correlation $(r>0.9)$ for $\mathrm{AD}(3.5 \mathrm{~m} / \mathrm{s}$ and individual speed) and AS $(3.5 \mathrm{~m} / \mathrm{s})$. Very large positive correlations $(0.7<r \leq 0.9)$ were found for all other comparisons of $t$ _evVel_Gonio and $t$ _evVel_Gyro. Regression analysis revealed that t_evVel_Gyro was strongly related to t_evVel_Gonio at all velocities and all footwear conditions $(p<0.001)$.

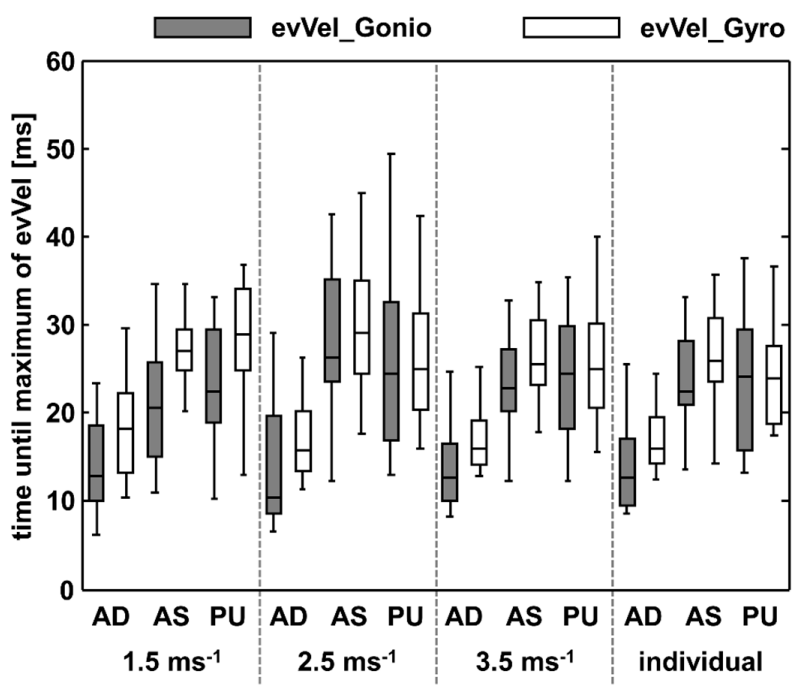

Figure 2. Boxplot of time until evVel_Gonio (t_evVel_Gonio, grey) and time until evVel_Gyro (t_evVel_Gyro, white) for the four velocities and three footwear conditions: Adidas AdiStar (AD); Asics Gel Nimbus 12 (AS); PUMA Speed 600 (PU). 
Table 3. Mean differences (MD) and standard deviations (SD) between t_evVel_Gonio and t_evVel_Gyro of the four velocities and the three footwear conditions: Adidas AdiStar (AD), Asics Gel Nimbus 12 (AS), PUMA Speed 600 (PU); Pearson's correlation coefficient was interpreted as trivial $(r \leq 0.1)$, small $(r \leq 0.3)$, moderate $(r \leq 0.5)$, large $(r \leq 0.7)$, very large $(r \leq 0.9)$, and extremely large $(r>0.9)$.

\begin{tabular}{cccccc}
\hline Speed & Footwear Condition & MD $(\mathbf{m s})$ & SD $(\mathbf{m s})$ & $\boldsymbol{r}$ & $\boldsymbol{R}^{\mathbf{2}}$ \\
\hline \multirow{3}{*}{$1.5 \mathrm{~m} / \mathrm{s}$} & AD & 4.1 & 2.4 & $0.88^{1}$ & $0.77^{2}$ \\
& AS & 5.2 & 4.4 & $0.78^{1}$ & $0.60^{2}$ \\
& PU & 5.3 & 3.5 & $0.86^{1}$ & $0.73^{2}$ \\
\hline \multirow{3}{*}{$2.5 \mathrm{~m} / \mathrm{s}$} & AD & 3.4 & 3.4 & $0.89^{1}$ & $0.77^{2}$ \\
& AS & 1.6 & 3.7 & $0.88^{1}$ & $0.76^{2}$ \\
& PU & 1.0 & 6.6 & $0.71^{1}$ & $0.49^{2}$ \\
\hline \multirow{3}{*}{$3.5 \mathrm{~m} / \mathrm{s}$} & AD & 3.2 & 2.0 & $0.91^{1}$ & $0.83^{2}$ \\
& AS & 2.8 & 2.2 & $0.92^{1}$ & $0.84^{2}$ \\
& PU & 1.9 & 4.3 & $0.83^{1}$ & $0.67^{2}$ \\
\hline \multirow{3}{*}{ Individual speed } & AD & 3.3 & 1.8 & $0.94^{1}$ & $0.87^{2}$ \\
& AS & 2.5 & 2.4 & $0.89^{1}$ & $0.79^{2}$ \\
& PU & 1.3 & 4.0 & $0.85^{1}$ & $0.72^{2}$ \\
\hline
\end{tabular}

${ }^{1}$ Pearson's correlation coefficient: $p<0.001 ;{ }^{2}$ Regression analyses: $p<0.001$.

\section{Discussion}

The purpose of the present study was to compare the accuracy of gyroscopes and electrogoniometers when measuring evVel and $\mathrm{t} \_$evVel.

The results of evVel revealed a small MD between $10.4 \pm 49.3$ and $49.8 \pm 46.9 \mathrm{deg} / \mathrm{s}$, which was lower than the difference reported by Brauner et al. [10]. They compared angular velocities in the frontal plane obtained from an optoelectronic system and a gyroscope and found an MD of $77.9 \mathrm{deg} / \mathrm{s}$. The greatest MD found in our study $(49.8 \pm 46.9 \mathrm{deg} / \mathrm{s})$ was distinctly lower than the repeatability coefficient found by Oriwol et al. [7] when investigating within-subject variability of the maximum eversion velocity $(169.0 \mathrm{deg} / \mathrm{s})$. This means that our MDs were within the limits of total measurement variability, and therefore were considered non-relevant. Furthermore, high standard deviations - in some cases higher than MD—show that there was also a high variability within the subjects. One reason could be the individual walking and running gait of the subjects, which can slightly influence the measurement of evVel_Gonio and evVel_Gyro. Additionally, footwear conditions and locomotion velocities can influence rearfoot motion of subjects $[4,5,9,12,22]$. In our study, the softest shoe (AS) showed the lowest evVel compared to the harder shoes (PU and AD). This is in line with previous findings $[4,5,23]$. While MD was similar for the footwear conditions during walking, there was a general trend of decreasing differences between evVel_Gyro and evVel_Gonio seen for all running velocities with decreasing footwear stiffness (AD > PU > AS) (Table 2 and Figure 1). MD was even negative for the softest shoe (AS), which means that higher evVel was measured with the electrogoniometer than with the gyroscope. It seems that MD is influenced by footwear conditions and locomotion velocities. We could not conclusively determine which measurement device was influenced by which conditions. However, as mentioned, MD was small and within the variability, and therefore, MD can be declared non-relevant, regardless of positive or negative values. Furthermore, Pearson's correlation coefficient showed a very large positive correlation of at least $r \geq 0.88$ for all tested footwear conditions and locomotion speeds between evVel_Gonio and evVel_Gyro. The correlations in our study are larger than those reported in previous studies comparing maximum angular velocities of gyroscopes with optoelectronic systems $[8,10,14]$. One reason for the lower correlations in the mentioned studies may be marker-artifacts due to wobbling during initial ground contact and the subsequent problematic foot model calculations by the respective software. In our study, both devices were attached firmly to the heel cap of the right shoe to minimize wobbling, 
and the angular velocities were measured at this position in the frontal plane directly. However, the flex arm of the goniometer-which was attached at the posterior lower leg-can be slightly influenced by skin displacement during initial ground contact, especially at higher velocities and with harder shoes. Furthermore, Brauner et al. [10], Shih et al. [8], and Lederer et al. [14] used lower sampling rates to compare optoelectronic systems and gyroscopes, resulting in less data, which could have influenced their results. In our study, data of both devices were recorded with a high sampling rate of $1000 \mathrm{~Hz}$, and an effect due to different or too low sampling rates can therefore be excluded. Due to the very large and extremely large positive correlations, and the strong relationships between evVel_Gyro and evVel_Gonio, we can confirm hypothesis H1.

T_evVel_Gyro was determined later than t_evVel_Gonio for all footwear conditions and all locomotion speeds. This offset differs between footwear conditions and locomotion speeds. Surprisingly, two trends were observed. First, the largest MD was found for the walking condition (for all three footwear conditions). Second, the hardest footwear condition (AD) revealed the largest $\mathrm{MD}$ and PU the lowest MD for all running velocities. Thus, this offset seems unrelated to rearfoot stiffness or Heel height $_{\text {, }}$ and another unknown factor must be influencing t_evVel_Gyro. In addition, very large and extremely large positive correlations as well as strong relationships of $t$ _evVel_Gyro and t_evVel_Gonio were found. Therefore, our hypothesis $\mathrm{H} 2$ can be confirmed.

One limitation of the current study is that only rearfoot strike runners were investigated. Further studies should also investigate midfoot and forefoot strikers. Especially during initial ground contact with the forefoot first, the changed orientation of the gyroscope can lead to a crosstalk of the sensitive axes. This can influence the subsequently derived maximum angular velocities.

In conclusion, the results of this study are necessary when interpreting peak angular velocities for different footwear conditions and locomotion speeds which were measured with a heel-mounted gyroscope in the frontal plane. The results of the present study showed strong relationships between evVel_Gyro and evVel_Gonio for locomotion at 1.5, 2.5, $3.5 \mathrm{~m} / \mathrm{s}$, and at individual speed, and for three different footwear conditions. Furthermore, strong relationships of t_evVel_Gyro and t_evVel_Gonio were found for all 12 conditions. Based on our results, it can be concluded that gyroscopes can accurately determine peak eversion velocity and time until peak eversion velocity during a variety of conditions (e.g., to compare the influence of footwear conditions on evVel in prolonged field runs).

Acknowledgments: The authors would like to thank Lisa Peterson for proofreading the paper as a native speaker. This research was supported by Puma ${ }^{\circledR}$ Inc., Germany. The publication costs of this article were funded by the German Research Foundation/DFG and the Technische Universität Chemnitz, Chemnitz, Germany, through the funding program Open Access Publishing.

Author Contributions: Christian Mitschke and Thomas L. Milani conceived and designed the experiments; Christian Mitschke performed the experiments; Christian Mitschke, Matthias Öhmichen and Thomas L. Milani analyzed the data; Thomas L. Milani contributed reagents/materials/analysis tools; Christian Mitschke, Matthias Öhmichen and Thomas L. Milani wrote the paper.

Conflicts of Interest: The authors declare no conflicts of interest. The funding sponsors had no role in the design of the study, in the collection, analyses, or interpretation of data, in the writing of the manuscript, or in the decision to publish the results.

\section{References}

1. Stacoff, A.; Reinschmidt, C.; Nigg, B.M.; van den Bogert, A.J.; Lundberg, A.; Denoth, J.; Stüssi, E. Effects of foot orthoses on skeletal motion during running. Clin. Biomech. 2000, 15, 54-66. [CrossRef]

2. Segesser, B.; Nigg, B.M. Insertionstendinosen am Schienbein, Achillodynie und Überlastungsfolgen am Fuss.-Ätiologie, Biomechanik, therapeutische Möglichkeiten. Orthopäde 1980, 9, 207-214. [PubMed]

3. Vtasalo, J.T.; Kvist, M. Some biomechanical aspects of the foot and ankle athletes with and without shin splints. Am. J. Sports Med. 1983, 11, 125-130. [CrossRef] [PubMed]

4. De Wit, B.; De Clercq, D.; Lenoir, M. The effect of varying midsole hardness on impact forces and foot motion during foot contact in running. J. Appl. Biomech. 1995, 11, 395-406. [CrossRef] 
5. Milani, T.L.; Hennig, E.M.; Lafortune, M.A. Perceptual and biomechanical variables for running in identical shoe constructions with varying midsole hardness. Clin. Biomech. 1997, 12, 294-300. [CrossRef]

6. O'Connor, K.M.; Hamill, J. The role of selected extrinsic foot muscles during running. Clin. Biomech. 2004, 19, 71-77. [CrossRef]

7. Oriwol, D.; Sterzing, T.; Milani, T.L. The position of medial dual density midsole elements in running shoes does not influence biomechanical variables. Footwear Sci. 2011, 3, 107-116. [CrossRef]

8. Shih, Y.; Ho, C.-S.; Shiang, T.-Y. Measuring kinematic changes of the foot using a gyro sensor during intense running. J. Sports Sci. 2014, 32, 550-566. [CrossRef] [PubMed]

9. Struzik, A.; Konieczny, G.; Grzesik, K.; Stawarz, M.; Winiarski, S.; Rokita, A. Relationship between lower limbs kinematic variables and effectiveness of sprint during maximum velocity phase. Acta Bioeng. Biomech. 2015, 17, 131-138. [CrossRef] [PubMed]

10. Brauner, T.; Sterzing, T.; Milani, T.L. Ankle Frontal Plane Kinematics Determined By Goniometer, Gyrometer and Motion Analysis System: A Measurement Device Validation. In Proceedings of the 22nd Congress of the International Society of Biomechanics, Cape Town, South Africa, 5-9 July 2009.

11. Liu, T.; Inoue, Y.; Shibata, K. Development of a wearable sensor system for quantitative gait analysis. Measurement 2009, 42, 978-988. [CrossRef]

12. Milani, T.L.; Schnabel, G.; Hennig, E.M. Rearfoot Motion and Pressure Distribution Patterns during Running in Shoes with Varus and Valgus Wedges. J. Appl. Biomech. 1995, 11, 177-187. [CrossRef]

13. Mayagoitia, R.E.; Nene, A.V.; Veltink, P.H. Accelerometer and rate gyroscope measurement of kinematics: An inexpensive alternative to optical motion analysis systems. J. Biomech. 2002, 35, 537-542. [CrossRef]

14. Lederer, P.; Blickhan, R.; Schlarb, H. RearfootAngle Velocities During Running-A Comparison Between Optoelectronic and Gyroscopic Motion Analysis. Port. J. Sport Sci. 2011, 11, 903-906.

15. Heidenfelder, J.; Sterzing, T.; Milani, T.L. Systematically modified crash-pad reduces impact shock in running shoes. Footwear Sci. 2010, 2, 85-91. [CrossRef]

16. Giandolini, M.; Horvais, N.; Farges, Y.; Samozino, P.; Morin, J.B. Impact reduction through long-term intervention in recreational runners: Midfoot strike pattern versus low-drop/low-heel height footwear. Eur. J. Appl. Physiol. 2013, 113, 2077-2090. [CrossRef] [PubMed]

17. Laughton, C.A.; McClay Davis, I.; Hamill, J. Effect of strike pattern and orthotic intervention on tibial shock during running. J. Appl. Biomech. 2003, 19, 153-168. [CrossRef]

18. Schwanitz, S.; Odenwald, S. Long-term cushioning properties of running shoes. Eng. Sport 2008, 7, 95-100. [CrossRef]

19. Schwanitz, S.; Möser, S.; Odenwald, S. Comparison of test methods to quantify shock attenuating properties of athletic footwear. Procedia Eng. 2010, 2, 2805-2810. [CrossRef]

20. Cohen, J. Quantitative Methods in Psychology. Psychol. Bull. 1992, 112, 155-159. [CrossRef] [PubMed]

21. Hopkins, W.G.; Marshall, S.W.; Batterham, A.M.; Hanin, J. Progressive statistics for studies in sports medicine and exercise science. Med. Sci. Sports Exerc. 2009, 41, 3-12. [CrossRef] [PubMed]

22. Lohman, E.B.; Sackiriyas, K.S.B.; Swen, R.W. A comparison of the spatiotemporal parameters, kinematics, and biomechanics between shod, unshod, and minimally supported running as compared to walking. Phys. Ther. Sport 2011, 12, 151-163. [CrossRef] [PubMed]

23. Nigg, B.M.; Bahlsen, H.A.; Luethi, S.M.; Stokes, S. The influence of running velocity and midsole hardness on external impact forces in heel-toe running. J. Biomech. 1987, 20, 951-959. [CrossRef]

(C) 2017 by the authors. Licensee MDPI, Basel, Switzerland. This article is an open access article distributed under the terms and conditions of the Creative Commons Attribution (CC BY) license (http://creativecommons.org/licenses/by/4.0/). 